\title{
Coledococele: Caso diagnosticado por resonancia magnética
}

\author{
Miguel Calderón H.', Jaime Abarzúa V.', Cosme Quiroga G.', Juan Gana A. ${ }^{2}$, Florencia de Barbieri M.. ${ }^{3}$
}

1. Interno de medicina. Escuela de medicina. Facultad de Medicina. Pontificia Universidad Católica de Chile, Santiago, Chile.

2. Departamento de gastroenterología. Facultad de Medicina. Pontificia Universidad Católica de Chile, Santiago, Chile.

3. Departamento de radiología. Facultad de Medicina. Pontificia Universidad Católica de Chile, Santiago, Chile.

\section{Choledococele: Case diagnosed by MRI}

\section{Resumen:}

El coledococele es una dilatación quística del segmento distal del conducto biliar común que sobresale en el lumen duodenal. Estos comprenden menos del $2 \%$ de todos los casos reportados de quistes de colédoco. Los hallazgos imagenológicos del coledococele y de los quistes de duplicación duodenal son muy similares, ambos presentan además clínica variada e inespecífica, por lo que el diagnóstico definitivo suele ser durante la cirugía. Presentamos un caso de una paciente de 10 años que consulta por dolor abdominal con elevación de transaminasas que, tras estudio con resonancia magnética con colangioresonancia y EDA se diagnostica coledococele.

Palabras clave: Colangiopancreatografía por RM; Coledococele; Quiste coledociano.

\begin{abstract}
:
Choledochocele is a cystic dilation of the distal segment of the common bile duct protruding into the duodenal lumen. Is also known as type III choledochal cyst according to Todani's classification. It is usually misdiagnosed as duodenal duplication cyst. Multislice spiral computed tomography and magnetic resonance cholangiopancreatography may be comparable to endoscopic retrograde cholangiography for diagnosis of choledochocele. We present a case of a 10-years-old girl presented with abdominal pain, elevation of transaminases and a magnetic resonance cholangiopancreatography (MRCP) scan that revealed a choledochocele.
\end{abstract}

Key words: Choledochocele; Choledochal cyst; Magnetic resonance cholangiopancreatography.

Calderón M., et al. Coledococele: caso diagnosticado por resonancia magnética. Rev Chil Radiol 2020; 26(2): 76-79. *Correo electrónico: Miguel Calderón / macalderon@uc.cl

Trabajo enviado el 04 de noviembre de 2019. Aceptado para publicación el 17 de marzo de 2020.

\section{Introducción}

El coledococele (también conocido como quiste de colédoco tipo III según la clasificación de Todani(1) es una dilatación quística del segmento distal del conducto biliar común que sobresale en el lumen duodenal. Considerados raros, estos casos comprenden menos del $2 \%$ de todos los casos reportados de quistes de colédoco ${ }^{(2)}$.

Los hallazgos imagenológicos del coledococele y de los quistes de duplicación duodenal son muy similares, ambos presentan además clínica variada e inespecífica, por lo que el diagnóstico definitivo suele ser durante la cirugía.

\section{Caso}

Presentamos un caso de paciente de sexo femenino de 10 años que consulta en atención primaria por cuadro de dolor abdominal con elevación de transaminasas en agosto del año 2018, con SGOT 1.6 veces el valor normal (VN) y SGPT 7 veces VN. 
Se realiza estudio para VHB, CMV y VEB, negativos. Sin antecedentes familiares relevantes.

Fue referida a atención terciaria en gastroenterología pediátrica. Al examen físico no presentaba alteraciones evidentes. Se continuó estudio, con persistencia de elevación de transaminasas (SGPT 6 veces VN y SGOT 1.1 veces VN), con relación SGPT/ SGOT de 3, elevación de GGT (3,6 veces VN) y FA (1.2 veces $\mathrm{VN}$ ), sin hiperbilirrubinemia ni alteración de función sintética hepática. Además, se descartaron causas de daño hepático con ceruloplasmina, cupremia, A1AT, VHE, VHA, ATG y Ac antiendomisio en valores normales.

Se complementó el estudio con una resonancia magnética con colangioresonancia que mostró una lesión quística que protruía hacia el lumen duodenal de $12 \times 7 \mathrm{~mm}$ en sus ejes mayores y que se encontraba en directa relación con la desembocadura del colédoco en la ampolla de Vater y discreta disminución de calibre del conducto hepático común que determinaba discreta dilatación de los conductos hepáticos derecho e izquierdo, sin compromiso de la vía biliar intrahepática (Figura 1). Posteriormente se realizó EDA que confirmó la presencia de un coledococele a nivel de ampolla mayor (Figura 2).

Se decide resolución endoscópica a través de CPRE para destechamiento de coledococele e instalación de stent plástico en vía biliar con evolución favorable.

\section{Discusión}

El coledococele (también conocido como quiste de colédoco tipo III según la clasificación de Todani ${ }^{(1)}$ corresponde al $1.4 \%$ de todas las dilataciones biliares ${ }^{(3)}$.

Sarris y Tsang ${ }^{(4)}$ propusieron una clasificación de los coledococele en tipo A (subclasificados en A1, A2 y A3 según si tienen entrada común del ducto pancreático y el conducto biliar común distal en el quiste, si tienen entradas separadas o si tienen entradas separadas pero son pequeños e intramurales, respectivamente) y tipo B (la ampolla vacía directamente en el duodeno y el quiste representa un divertículo del conducto biliar común distal protuyendo en el lumen duodenal).

Su etiología aún se desconoce. Algunos autores proponen alteraciones en la embriogénesis del conducto biliar(5), otros que podrían derivar de una estenosis papilar o disfunción del esfínter de Oddi podrían generar un aumento de presión del conducto biliar que terminaría en una evaginación al duodeno(6). Sarris y Tsang ${ }^{(4)}$ reportaron que hasta en un $63 \%$ de los casos se encontraría epitelio de mucosa duodenal. Histopatológicamente, la presencia de mucosa intestinal crea controversia sobre si el coledococele es un tipo de quiste de duplicación duodenal o una entidad única.

Los quistes de duplicación duodenales son también extremadamente raros, representando sólo entre el 5 al $10 \%$ de las duplicaciones del tubo digestivo ${ }^{(7)}$.

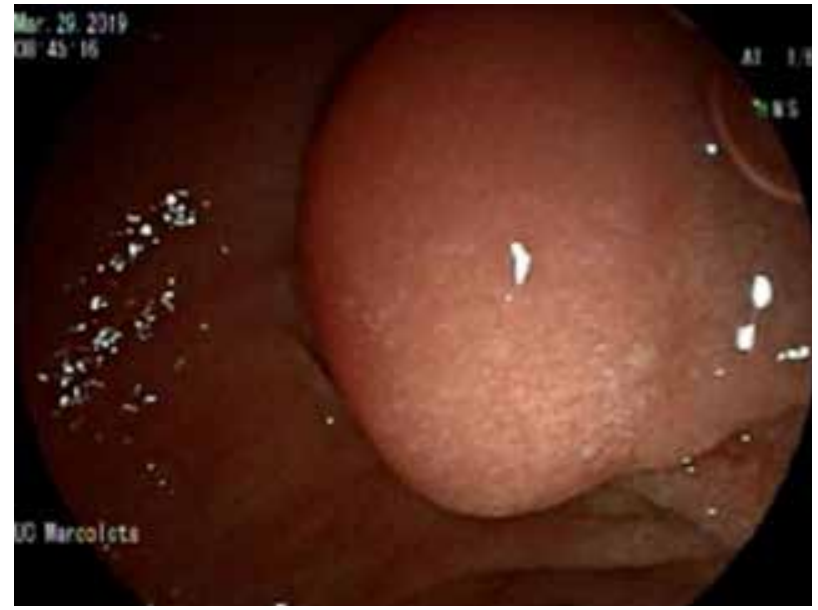

Figura 2: EDA en la segunda porción del duodeno, a nivel de la ampolla de Vater se observa un aumento de volumen de aproximadamente $1 \mathrm{~cm}$. de pared colapsable y fina con un pequeño orificio valvular, compatible con un coledococele.

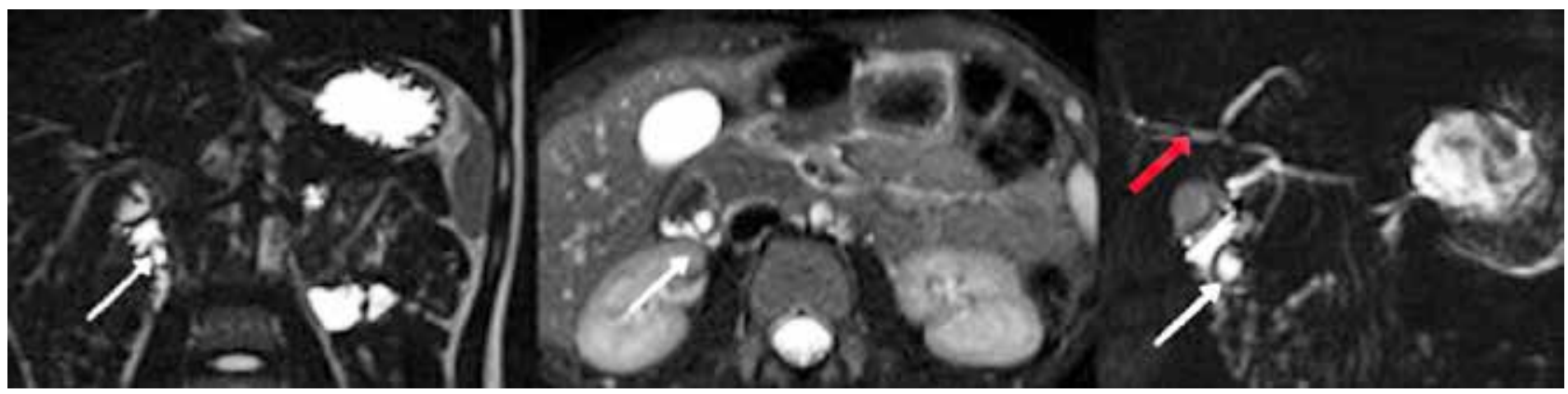

Figura 1: Se observa una imagen quística de paredes perceptibles, que se insinúa al lumen del duodeno, compatible con dilatación quística del colédoco distal (flechas blancas). No hay dilatación de la vía biliar ni del páncreas (flecha roja). 
Los coledococele y las duplicaciones quísticas duodenales tienen presentaciones clínicas similares: dolor abdominal, náuseas, vómitos, pancreatitis, colestasia, ictericia e infección quística. El examen de imagen juega un papel importante en el diagnóstico. El examen no invasivo con ultrasonido puede detectar una masa quística en la cavidad duodenal; sin embargo, la visualización se encuentra limitada por la presencia de asas intestinales.

Los estudios baritados del tracto gastrointestinal alto asociados a la colangiografía pueden ser de ayuda para diferenciar un coledococele de un quiste de duplicación duodenal, ya que los primeros captan contraste en la colangiografía pero no bario, mientras que la falta de captación en ambos estudios junto a la ausencia de contenido biliar en los quistes definen las duplicaciones duodenales ${ }^{(8)}$.

En las endoscopías digestivas altas, la papila siempre se encuentra proximal a los quistes por duplicación duodenal, mientras que en los coledococele se encuentran a distal salvo en los tipo B.

Los hallazgos en la resonancia magnética con estudio de colangioresonancia para ambos casos es la presencia de una lesión quística en íntima relación la segunda porción del duodeno y en ocasiones puede ser difícil distinguirlas sólo con clínica y con imagenología habitual( ${ }^{(9)}$. Un coledococele es definido por su relación con el conducto biliar, mientras que el quiste de duplicación duodenal está definida por su relación con el duodeno y por su pared de mayor grosor, similar a la pared del intestino adyacente. En la literatura, la CPRE se ha realizado en el 67\% de los casos notificados, y este método tiene el beneficio de la implementación simultánea del tratamiento aunque se considera que la tomografía computarizada multicorte y la colangiopancreatografía por resonancia magnética tienen especificidad y sensibilidad comparables, y pueden reemplazar a la CPRE para el diagnóstico ${ }^{(10,11,12)}$.

El tratamiento quirúrgico abierto, como la extirpación completa del quiste transduodenal con esfinteroplastía, así como la esfinterotomía endoscópica son tratamientos viables que se han aplicado en pacientes pediátricos con éxito ${ }^{(13,14)}$.

En conclusión, el coledococele es una anomalía rara, más aún en la edad pediátrica y difícil de sospechar por la clínica aislada. El método diagnóstico de elección es la resonancia magnética de abdomen con colangioresonancia y el método de tratamiento aún es controvertido.

\section{Referencias}

1. Todani T, Watanabe Y, Narusue M, Tabuchi K, Okajima K. Congenital bile duct cysts: Classification, operative procedures, and review of thirty-seven cases including cancer arising from choledochal cyst. Am J Surg. 1977; 134: 263-269.

2. Yamaguchi M. Congenital choledochal cyst. Analysis of 1,433 patients in the Japanese literature. Am J Surg. 1980; 140: 653-657.

3. Congenital choledochal cyst. Analysis of 1,433 patients in the Japanese literature. Yamaguchi M Am J Surg. 1980 nov; 140(5): 653-657.

4. Sarris GE, Tsang D. Choledochocele: case report, literature review, and a proposed classification. Surgery. 1989 mar; 105(3): 408-414.

5. Tanaka T. Pathogenesis of choledochocele. Am J Gastroenterol. 1993; 88: 1140.

6. Sterling JA. Diverticula in the terminal portion of the common bile duct. Am J Pathol. 1949; 25: 325-337.

7. Hocking M, Young DG. Duplications of the alimentary tract. Br J Surg 1981; 68: 92-96.

8. Law R, Topazian M. Diagnosis and treatment of choledochoceles.. Clin Gastroenterol Hepatol. 2014 feb; 12(2): 196-203.

9. Koffie RM, Lee S, Perez-Atayde A, Mooney DP. Periampullary duodenal duplication cyst masquerading as a choledochocele. Pediatr Surg Int. 2012 oct; 28(10): 1035-1039.

10. Groebli Y, Meyer JL, Tschantz P. Choledochocele demonstrated by computed tomographic cholangiography: report of a case. Surg Today. 2000; 30: 272-276.

11. Park DH, Kim MH, Lee SK, Lee SS, Choi JS, Lee YS, et al. Can MRCP replace the diagnostic role of ERCP for patients with choledochal cysts? Gastrointest Endosc. 2005; 62: 360-366.

12. Sacher VY, Davis JS, Sleeman D, Casillas J. Role of magnetic resonance cholangiopancreatography in diagnosing choledochal cysts: case series and review. World J Radiol. 2013; 5(8): 304-312. doi:10.4329/wjr. v5.i8.304

13. Mane S, Arlikar J, Dhende N. Choledochocele: An unusual presentation in a premature neonate. J Indian Assoc Pediatr Surg. 2012; 17: 28-30.

14. Saeki I, Takahashi Y, Matsuura T, Takahata S, Tanaka $M$, Taguchi T. Successful endoscopic unroofing for a pediatric choledochocele. J Pediatr Surg. 2009; 44: 1643-1645. 
